\title{
CH. S. PEIRCE Y LA TEORÍA LITERARIA
}

\author{
José Domínguez Caparrós
}

(UNED, Madrid)

La reunión de estos días en Segovia, centrada en el pensamiento de Charles S. Peirce, no es más que un dato más que demuestra el hecho evidente de que la obra del filósofo norteamericano ha adquirido la propiedad de clásica. Por esto, sus escritos se estudiarán y comentarán constantemente en el ámbito de la ciencia o disciplina semiótica; y en cuanto clásicas, sus ideas adquieren un carácter general, intemporal, que las hace interesantes, hasta el punto de dotarlas de un sentido profético, que se cumple en cuanto que sirven para garantizar algunas soluciones a cuestiones que hoy nos preocupan.

Está claro, pues, que lo que yo voy a hacer es manipular algunas ideas de Peirce para comentar algo de lo que pasa en la teoría literaria actual. Cuando pienso en una manipulación para lo que yo voy a hacer es porque quiero vacunarme contra la más seria objeción que puede hacerme todo entendido en semiótica peirceana; pues está claro que yo no tengo que aportar gran cosa al descubrimiento del sentido de la filosofía del 
americano --me está vedado el placer de ir siguiendo el riquísimo encaje de las piezas de su razonamiento-. Pero, como profesional de la teoría literaria, pertenezco a esa especie de estudioso de la literatura que acude a la filosofía, y a tantos otros sitios, en busca de una mejor comprensión del fenómeno de la palabra artística. Es inevitable, pues, un contacto con el pensamiento de Peirce. Yo lo tuve de forma más directa cuando me vi obligado a tratar de caracterizar la poesía desde la semiótica; sobre esto volveré.

Una dificultad que se añade a las que encuentra el teórico de la literatura que quiere acercarse a Peirce es que éste es más un filósofo interesado en la lógica que en la estética; por esto, frecuentemente hay que hacer una transposición de lo que dice al campo de la estética, y de esta forma empezamos ya a producir nosotros mismos metáforas, sentidos.

\section{II}

Voy a recordar algunas cosas bien conocidas de todos, pero es que son necesarias en mi discurrir. El objeto de la semiótica es el proceso de creación de sentido en el texto, que se manifiesta como signo (Bobes Naves 1989: 101). Pero este signo es analizado de manera bien distinta en las dos tradiciones mayores de la semiótica a que solemos referirnos: la saussureana y europea, en general, por un lado; y la que arranca de Peirce y crece en suelo americano, por otro; la primera, de vocación linguística, se ve abocada a ser una teoría del texto; la segunda, de raíz lógica, es una filosofía abierta a consideraciones pragmáticas. Por lo que se refiere a la poesía, por ejemplo, a partir de la semiología el estudio de la misma queda caracterizado por unas constantes, que podemos enumerar de la siguiente forma: 1.- La linguiística dirige el estudio de la poesía, y la semiótica poética utiliza como conceptos básicos los de signo (y los con él relacionados de forma, sustancia, expresión y contenido), paradigma y sintagma, denotación y connotación, por citar los más llamativos. 2.- El modelo del signo lingüístico sirve casi siempre de marco de organización de los estudios de la poesía, en los que se suele distinguir un plano de la expresión y un plano del contenido, o un significante y un significado como ejes organizadores de todo el análisis. 3.- Como lenguaje especial, es decir, como semiótica o sistema semiótico peculiar, la poesía se caracteriza por ser el poema un signo complejo en el que se da una estrecha dependencia, un isomorfismo, entre significante y significado, o expresión y contenido; el signo poético, se dice, está motivado, es decir, establece una relación de semejanza entre el signo y la cosa de- 
signada, lo que significa; y por eso, a todo, en poesía, se le busca un significado.

Éstas son características de la poesía como lenguaje que se desprenden de la forma en que la estudian los modelos semiológicos que dependen estrechamente de la lingüística europea formulada por Saussure y Hjelmslev.

Si hoy la semiótica que arranca de Peirce es hegemónica, me parece que es porque integra en su concepción de la semiosis elementos de mayor alcance que los simplemente textuales o linguísticos a que frecuentemente queda reducida la semiología saussureana. Hoy, en efecto, se nos presenta mucho más próxima a las cuestiones que nos preocupan una definición como la que da Peirce de semiótica o de semiosis. La primera consiste en

la doctrine de la nature essentielle et des variétes fondamentales de semiosis possibles. (5.488) 1

¿Y qué es la semiosis?

Mais par "semiosis", j'entends, au contraire, une action ou influence qui est ou implique la coopération de trois sujets, tels qu'un signe, son objet et son interprétant, cette influence tri-relative n'étant en aucune façon reductible à des actions entre paires. (5.484)

\section{III}

Puesto que me está vedado el placer del conocimiento detallado del pensamiento de $\mathrm{Ch}$. S. Peirce, me siento igualmente relevado de la responsabilidad de ser un fiel intérprete del mismo. Tampoco es este mi propósito, sino que lo que yo pretendo es ofrecer a los especialistas en la seimótica peirceana las muestras de ciertos ecos de tal pensamiento en algunos de los postulados de la teoría literaria que parece haber detentado la hegemonía en la historia reciente de esta disciplina en algunas partes del mundo, al menos. Ahí van las muestras.

Primera: En su magnífico panorama de la crítica norteamericana después de la «nueva crítica», Frank Lentricchia comenta el trabajo de

1 Cito por Charles S. Peirce (1978). 
Eugenio Donato presentado en la famosa reunión de 1966 en la Johns Hopkins University que marca la entrada de Jacques Derrida en EE UU. El trabajo de Donato, en la traducción castellana - traducción sorprendentemente temprana, en 1972, cuando la edición inglesa es de 1970, y por eso abocada a pasar inadvertida prácticamente- , se titula «Las dos formas de expresión de la crítica». Lentricchia dice del mismo que allí se plantean las dos cuestiones esenciales que durante los años setenta van a obsesionar tanto a los derridianos como a los antiderridianos de Norteamérica: la primera es la cuestión del descentramiento o «estructuralidad de la estructura», es decir, la desaparición de un origen, de un punto exterior al texto que le sirviera de frontera al juego del significante lingüístico. Leo el extenso pasaje en que Lentricchia resume la segunda cuestión:

En segundo lugar, está la afirmación (implícita en algún pasaje de la obra estructuralista de Barthes, pero llevada a su punto crítico por Derrida) de que toda interpretación de una cadena de significantes resulta ser otra cadena de significantes, y nada más. A la luz de estos planteamientos es imposible otorgar al discurso crítico la consideración de «transparente» (ni siquiera la mera consideración de representación mimética); y, por consiguiente - escribe Donato-, tampoco se puede trazar una línea de «separación esencial entre literatura y crítica»: «cada signo es, en sí mismo, no cosa que se ofrece a la interpretación, sino interpretación de otros signos. Nunca se da un interpretandum que no sea ya interpretans, de modo que la interpretación queda necesariamente vinculada al ejercicio de la violencia y a la explicación». Donato añade a continuación algo que pronto sería recogido en los debates postestructuralistas: «sin duda alguna», esta idea «tendríamos que haberla captado ya en Nietzsche y Freud». A lo cual cabría añadir que en los Estados Unidos habríamos podido extraerla de la semiótica de Charles S. Peirce. (F. Lentricchia 1980: 158).

La última frase de la larga cita de F. Lentricchia es la que me interesa especialmente, por cuanto que ahí se encuentra expresado un intento de involucrar el pensamiento de Peirce en una cuestión tan importante como la forma de concebir la interpretación en buena parte de la crítica literaria más innovadora: serie infinita de signos. Véase Roland Barthes y su idea de la crítica como interpretación, no de un sentido verdadero, sino de cadenas de símbolos producidos por la obra; el crítico no hace más que continuar las metáforas de la obra, y, desde este momento, su quehacer es tan creador como el del escritor (R. Barthes 1966: 67-74) 22. Peirce, por lo demás, se ve llevado a la compañía de los dos máximos representantes de la hermenéutica de la desconfianza, según P. Ricoeur.

2 La crítica a que Dan Sperber somete las prácticas interpretativas del mito -las que buscan un sentido oculto, como las psicoanalíticas- recuerda el funcionamiento del interpretante en el modelo peirceano. En efecto, según Sperber, estas explicaciones del símbolo no hacen una sustitución del segundo término al primero, sino que llevan a cabo una extensión, un desarrollo del símbolo (1978: 73 y ss). Para Dan Sperber, las interpretaciones semiológicas de los mitos no son tales, sino simples extensiones. Las palabras con que termina su crítica a la exégesis y al psicoanálisis son claras: «Aquí reside precisamente su capacidad simbólica: el inevitable fracaso de todas las tentativas les asegura al mismo tiempo la multiplicación. Así, las tentativas exegéticas y 
Paul de Man - y ésta es la segunda de las muestras prometidas - se ve atraído por la concepción de una retórica pura que, como es sabido, está relacionada con el interpretante del modelo de signo peirceano, y que al crítico americano lo reafirma en su idea de la retórica como figura o tropo intralingüístico. Dice Paul de Man:

Para Peirce, la interpretación de un signo no es un significado, sino otro signo; es una lectura, no una descodificación, y esta lectura, a su vez, hà de ser interpretada por otro signo, y así ad infinitum (1979: 22).

Parece que hay materia para un trabajo, si es que no está ya hecho, acerca de la presencia de Peirce en la moderna teoría literaria norteamericana. Esta presencia no excluye la posibilidad de una mala interpretación. Pues una mala lectura, conscientemente metafórica, del pensamiento de Peirce por parte de Harold Bloom es la aplicación que éste hace de la idea de triplicidad del signo semiótico al modelo del poema:

Aplicando el principio de la mala captación (misprision), traslademos a Peirce al lenguaje de la poesía. El poema es una idea de Triplicidad, una relación triádica, porque el signo es el nuevo poema, su objeto es el texto precursor (por múltiple o imaginario que sea) y el pensamiento que interpreta es la lectura del poema, aunque esta lectura sea ya por sí misma un signo. (Apud F. Lentricchia 1980: 312)

Sea ésta una tercera muestra.

Yo ahora pongo sobre la mesa algunos fragmentos del pensamiento de Peirce sobre el signo - y especialmente sobre el interpretante- por si los especialistas en semiótica creen justificado el establecimiento de un

psicoanalíticas parecen responder a un proyecto cultural: en apariencia, interpretar el simbolismo; de hecho, renovarlo, porque toda clase de símbolos forma parte del simbolismo mismo» (1978: 76).

Umberto Eco, que vincula el trabajo desarrollado en alguna de sus obras (Tratado de semiótica general, Lector in fabula, Semiótica y filosofía del lenguaje) con la idea peirceana de semiosis ilimitada, se desmarca explícitamente de las repercusiones que tal idea haya podido tener en la deconstrucción. El enunciado de su postura más reciente no ofrece dudas: «La teoría peirceana de la semiosis ilimitada no puede llevar a sostener, como ha hecho Derrida, una teoría de la interpretación como deriva y deconstrucción. Los textos tienen un sentido, incluso cuando los sentidos son muchos; lo que no puede decirse es que no existe ninguno, o que todos sean igualmente buenos. El texto interpretado impone unas restricciones a sus intérpretes. Los límites de la interpretación coinciden con los derechos del texto (lo que no quiere decir que coincidan con los derechos de su autor)» (1991: 9). 
parentesco o un aval para la moderna concepción de la crítica como producción ilimitada de signos.

La muy conocida definición del signo (2.228) y la clasificación de las ramas de la semiótica según se refieran a cada uno de los componentes del mismo signo (2.229), ofrecen elementos para una comparación, como son: el que el interpretante es un signo que está en la mente de aquel a quien se dirige el representamen; o que la rama de la semiótica relacionada con el interpretante es la retórica pura, cuyo

trabajo es averiguar las leyes de cada inteligencia científica: un signo da nacimiento a otro y, especialmente, un pensamiento produce otro pensamiento ${ }^{3}$.

$\mathrm{Si}$ el interpretante es un signo, lógicamente tiene que tener, a su vez, un interpretante que será otro signo. Esto queda claro en la forma en que Peirce caracteriza al signo en el párrafo 2.303:

Cualquier cosa que determina alguna otra (su interpretante) para que se refiera a un objeto al cual él mismo se refiere (su objeto); de la misma manera el interpretante se convierte a su vez en un signo, y así ad infinitum.

El interpretante, por lo que sigue en este mismo párrafo, debe ser formulado en una interpretación, en algún signo externo, porque, si no, «resulta imposible descubrir que alguna vez existió tal idea en esta conciencia».

3 Cito por Charles S. Peirce, 1987. Recordemos la definición exacta de signo:

Un signo o representamen es algo que representa algo para alguien en algún aspecto o carácter. Se dirige a alguien, es decir, crea en la mente de esa persona un signo equivalente o, quizás aún, más desarrollado. A ese signo creado, yo lo llamo el Interpretante del primer signo. (2.228)

Las otras dos ramas de la semiótica, junto a la retórica pura, son la gramática especulativa y la lógica -que trata del objeto-. Véase el comentario que hace Gérard Deledalle a la definición de signo, y el lugar del interpretante:

Le signe interprétant renvoie lui-même syntactiquement à un signe interprétant en une série infinie d'interprétants qu'interrompt pragmatiquement et provisoirement, selon les situations existentielles, l'interprétant logique ultime: l'habitude.

(1978: 229) 
La pregunta siguiente es: ¿pero cuándo terminamos de interpretar, de producir signos? Cuando pragmáticamente la costumbre establece el significado lógico definitivo. La costumbre - desechados los conceptos, los deseos y las expectativas como las otras clases de categorías de los hechos mentales que tienen una referencia general- se convierte en la esencia del interpretante lógico (Écrits 5.486). Bien claro lo dice en el párrafo 5.491:

\footnotetext{
L'habitude formée délibérément par analyse d'elle-même - parce que formée à l'aide des exercices qui la nourrissent - est la définition vivante, l'interprétant logique véritable et final. Par suite, pour exprimer le plus parfaitement possible un concept que les mots peuvent communiquer, il suffira de décrire l'habitude que ce concept est calculé à produire».
}

¿Idea de la interpretación como práctica perlocutiva — cálculo de producción de una costumbre-; o es la interpretación un efecto de la costumbre, y podría, entonces, relacionarse la costumbre con las constricciones de una comunidad interpretativa al estilo de Stanley Fish? Son éstas preguntas que hay que plantear más bien para una discusión entre especialistas en la semiótica de Peirce. Sí puedo adelantar que en ninguno de los dos libros en que se recogen la mayoría de los trabajos de S. Fish $(1980,1989)$ aparece, en los índices, el nombre de Peirce.

\section{V}

La discusión podría prolongarse en la redefinición que hace Ch. Morris del signo. Sabido es que añade un cuarto factor, el intérprete - ya diferenciado del interpretante de Peirce-. Señala Morris (1938: 27-28) en el proceso semiótico la implicación de los siguientes componentes: el vehículo sígnico, el designatum, el interpretante y el intérprete. Por ejemplo: en el caso del perro que al oír un sonido determinado caza ardillas tenemos el sonido como vehículo sígnico (S), el significado de cazar ardillas es el designatum (D), y la conducta que responde a esa acción es el interpretante (I); los intérpretes son los agentes del proceso, los usuarios de los signos. Ahora bien, en el epígrafe siguiente, cuando se trata de los niveles de semiosis, fundados en la relación diádica de cada uno de los tres correlatos (vehículo, designatum e intérprete) de la semiosis con el signo, sorprendentemente ha desaparecido el interpretante, ha desaparecido, pues, la posibilidad de interpretación interna al signo: 
En términos de los tres correlatos (vehículo sígnico, designatum, intérprete) de la relación triádica de semiosis, pueden abstraerse - para convertirse en objeto de estudio-- una serie de relaciones diádicas. (1938: 31)

Como es bien sabido, estas relaciones son las que constituyen las tres ramas de la semiótica: sintaxis, semántica y pragmática ${ }^{4}$. Lo que me parece apreciar en el modelo de $\mathrm{Ch}$. Morris es una simplificación que va acompañada de una normalización que trata de objetivar todo el proceso en una exterioridad. En este sentido, si bien la propuesta de Morris es la que ha tenido más fortuna en la división de tareas semióticas de los estudios literarios, me parece que la de Peirce sigue siendo más sugerente, menos tranquilizadora. Pero yo no quiero ir más allá en la interpretación del pensamiento semiótico.

Como se trata de proponer ideas para una discusión, no quiero ocultar que hay también algún estudio sobre el concepto de la interpretación en Ch. Sanders Peirce que descalificaría cualquier propuesta como la que subyace en todo lo dicho hasta ahora. Imagino que se puede ilustrar con comentarios de la teoría peirceana que en absoluto apoyarán la supuesta relación establecida por Lentricchia o por Paul de Man. Yo conozco uno de estos comentarios y lo voy a exponer. Se trata del artículo publicado por Hanna Buczynska-Garewicz en la revista Versus (n. $\left.{ }^{\circ} 49,1988\right)$ sobre la semiótica y el arte de comprender. En realidad se trata de una glosa y comentario a la teoría de Peirce sobre el signo, cuya semiótica concibe la autora como un teoría de la interpretación 5 . Un resumen que quisiera dar cuenta de este trabajo recogería ideas bien conocidas y ya comentadas, como: que el signo es un pensamiento y apela a otro pensamiento para interpretarlo; que el signo está inextricablemente unido a la interpretación; que la interpretación semiótica es un proceso sin fin de producción de signos; que este proceso se para cuando se establece la relación con el mundo objetivo; lo que le da un carácter no subjetivo, según la autora, quien resalta la importancia de la costumbre como máxima pragmática;

4 Parece que en el pensamiento de Morris puede apreciarse algún cambio en su concepción de la pragmática, como observa Carmen Bobes (1989: 99): primero se define como la relación con sus intérpretes, «y más tarde añadirá a esa relación con los usuarios, las de los signos con la situación de uso a través de todas las circunstancias que participan en él». Pienso que la ambigüedad en la caracterización del signo, por parte de Morris, no es ajena a tal duplicidad.

5 Se basa fundamentalmente en Peirce también Alberto Álvarez Sanagustín en su trabajo sobre la interpretación creativa (1990). 
por último, que la interpretación semiótica de Peirce no tiene en cuenta el sentido histórico. Pues bien, después de la breve síntesis, llega a la conclusión de que

semiotics stays in a crucial opposition to many of the present tendencies in the theory of interpretation. (1988: 63)

Y cita la autora, entre las que tiene en su mente, las siguientes: el historicismo, el irracionalismo y voluntarismo o el antilogocentrismo (es decir, la deconstrucción), el relativismo cultural y emocional de la semiótica étnica, y, finalmente, el psicologismo y behaviorismo. En final epigramático, dice Hanna Buczynska-Garewicz:

Apparently, Peirce's notion of interpretation does not belong to the late 20th century -which does not, however, imply that Peirce was wrong. (1988: 63)

Estas palabras dan una de las respuestas posibles a la pregunta con que estuve tentado de empezar mis palabras: ¿es Peirce uno de los responsable de la deconstrucción? Otra respuesta sería decir que efectivamente es uno de los responsables, como podría serlo todo estructuralista coherente con su pensamiento, empezando por Saussure, pues no olvidemos que lo más descentrado es la estructura.

\section{Referencias bibliográficas}

Álvarez SANAGUSTín, A. (1990): «La interpretación creativa”. En Investigaciones Semióticas. III. Retórica y lenguajes, 145-154. Madrid: UNED.

BARTHES, R. (1966): Critique et Verité. Paris: Seuil.

Bobes Naves, M. C. (1989): La semiología. Madrid: Síntesis.

BUCZYNSKA-GAREWICZ, H. (1988): «Semiotics and art of understanding". Versus 49, 59-63.

DE MAN, P. (1979): Alegarías de la lectura. Traducción de Enrique Lynch. Barcelona: Lumen, 1990.

Eco, U. (1991): «Los límites de la interpretación”. Revista de Occidente 118, 5-24.

FISH, S. (1980): Is there a text in this class? Cambridge, Mass.: Harvard U. P.

- (1989): Doing what comes naturally. Oxford: Clarendon Press.

Lentricchia, F. (1980): Después de la 'nueva crítica'. Traducción de Ramón Buenaventura. Madrid: Visor, 1990. 
MACKSEY, R., Donato, E. (eds.) (1970): Los lenguajes críticos y las ciencias del hombre. Traducción de José Manuel Llorca. Barcelona: Barral, 1972.

MorRIs, CH. (1938): Fundamentos de la teoría de los signos. Traducción de Rafael Grasa. Barcelona: Paidós, 1985.

PEIRCE, CH. S. (1978): Écrits sur le signe. Rassemblés, traduits et commentés par Gérard Deledalle. Paris: Seuil.

- (1987): Obra lógico-semiótica. Armando Sercovich (ed.). Madrid: Taurus.

SPERBER, D. (1978): El simbolismo en general. Traducción de J. M. García de la Mora. Barcelona: Anthropos, 1988. 